\title{
Design and Testing of an Underwater Microscope with Variable Objective Lens for the Study of Benthic Communities
}

\author{
Kamran Shahani ${ }^{1} \cdot$ Hong Song ${ }^{1} \cdot$ Syed Raza Mehdi ${ }^{1}$. Awakash Sharma ${ }^{2} \cdot$ Ghulam Tunio $^{2} \cdot$ Junaidullah Qureshi $^{1}$. \\ Noor Kalhoro ${ }^{3} \cdot$ Nooruddin Khaskheli $^{3}$
}

Published online: 2 March 2021

(C) The Author(s) 2021

\begin{abstract}
Monitoring the ecology and physiology of corals, sediments, planktons, and microplastic at a suitable spatial resolution is of great importance in oceanic scientific research. To meet this requirement, an underwater microscope with an electrically controlled variable lens was designed and tested. The captured microscopic images of corals, sediments, planktons, and microplastic revealed their physical, biological, and morphological characteristics. Further studies of the images also revealed the growth, degradation, and bleaching patterns of corals; the presence of plankton communities; and the types of microplastics. The imaging performance is majorly influenced by the choice of lenses, camera selection, and lighting method. Image dehazing, global saturation masks, and image histograms were used to extract the image features. Fundamental experimental proof was obtained with micro-scale images of corals, sediments, planktons, and microplastic at different magnifications. The designed underwater microscope can provide relevant new insights into the observation and detection of the future conditions of aquatic ecosystems.
\end{abstract}

Keywords Underwater microscope $\cdot$ Optics $\cdot$ Corals $\cdot$ Sediments $\cdot$ Planktons $\cdot$ Microplastic $\cdot$ Arduino

\section{Introduction}

Over the past few years, underwater microscopic imaging has drawn the attention of researchers toward the study of marine ecosystems, corals, kelp forests, mangroves, algae, and millimeter- to micrometer-scale bed sediments. The areas of

\section{Article Highlights}

- Significance of in situ microscopy in aquatic ecosystems is discussed.

- An underwater microscope is designed and tested for the observation of the aquatic ecosystem.

- Coral bleaching, zooplankton, sediments, and microplastics are analyzed to verify the feasibility and imaging performance of the underwater microscope.

- Experiments prove that the underwater microscope is reliable for underwater ecosystem observation.

Hong Song

hongsong@zju.edu.cn

1 Ocean Engineering and Technology, Ocean College, Zhejiang University, Zhoushan 316000, China

2 Marine Science, Ocean College, Zhejiang University, Zhoushan 316000, China

3 Port and Coastal offshore Engineering, Ocean College, Zhejiang University, Zhoushan 316000, China interest related to underwater microscopic imaging include the detection of algae patterns in coral bleaching and the determination of sediment grain size (Mccook et al. 2001; Rubin 2004; Gao and Collins 1994). The microscopic analysis of plankton and microplastics has been an active research area in the past years. These studies are useful for geological and biological applications. Biological and sedimentological processes occurring in an artificial environment may vary from those occurring in natural environments in terms of temperature, density, carbon dioxide, turbidity, $\mathrm{pH}$, sedimentation, and mechanical disturbance (Ateweberhan et al. 2013; Delgadillo-Nuño et al. 2014). As a result, in the research on the benthic community temporal growth, it is difficult to study the biological and sedimentological processes in the laboratory, because of the problem of sampling site alteration (Schutte et al. 2010). Other approaches employed involve the use of holography and a long-distance fixed objective lens to acquire the microscopic images of the benthic aquatic floor (Akiba and Kakui 2000; Arora and Sahoo 2015; Jaffe et al. 2001; Kennelly and Underwood 1984; Mullen et al. 2016; Neushul 1972; Talapatra et al. 2013). Although the previously adopted techniques were practical, they lack integrated variable magnification, deployment methods, and microscope positioning for particular focus. 
As a result, in situ microscopy is an excellent technique for studying the microscopic species in aquatic ecosystems. In this paper, we present a novel method for in situ microscopy. The design comprises an imaging and control unit in a compact container, which allows for the system usage as an autonomous underwater vehicle or a remotely operated vehicle mount. A two-axis stepper motor-controlled mechanism allows the necessary movement of the microscope to bring the target in focus.

\section{Methodology}

\subsection{Microscope Design and Operation}

The designed underwater microscope (Figure 1a-d) has two units: a control unit that holds the operator interface to regulate lighting and magnification level and an imaging unit that holds the electronic parts, camera, one string of led lights, and optical parts. The design and working principles of the underwater microscope are presented in detail herein.

The housing of the control and imaging unit is made of a polymethyl methacrylate, which lingers on the water surface. The imaging unit is optically transparent and can bear the pressure corresponding to $100-\mathrm{m}$ depth. This allows for high-depth imaging, since the entire optical paths are in the air through the house rather than water. The underwater microscope has manual- and auto-focus capabilities, due to a motor-driven variable objective lens that provides different magnifications and working distances through an optical window when the user smoothly moves the microscope head. Magnification can be expanded or reduced by adjusting the level of the objective lens from $\times 0.7-4.5$. One variable objective lens, two Barlow lenses, one extension tube or adaptor, one charge-coupled device (CCD) camera, and one screen were used to produce a total magnification of $\times 1542.6$ in this study. This magnification could be used for the study of most corals, bed sediments, planktons, and microplastics. The focusing and magnification can be controlled via buttons and knobs attached to the control unit. The images captured by the underwater microscope, with a CCD camera in the imaging unit, were recorded on a memory card installed with a WiFi module. This allows the download of images upon returning to the surface via a wireless link or through a tether cable attached to the underwater microscope.

\subsubsection{Microscope Optics and Electronics}

The control and imaging unit are connected through a 30-m CAT5e cable. The imaging system contains an Arduino Nano microcontroller, 64-gigabyte memory card, HDMI-to-RJ45 video converter, power regulators, battery charge controller, DC motor, light controller, and lithium-ion batteries.

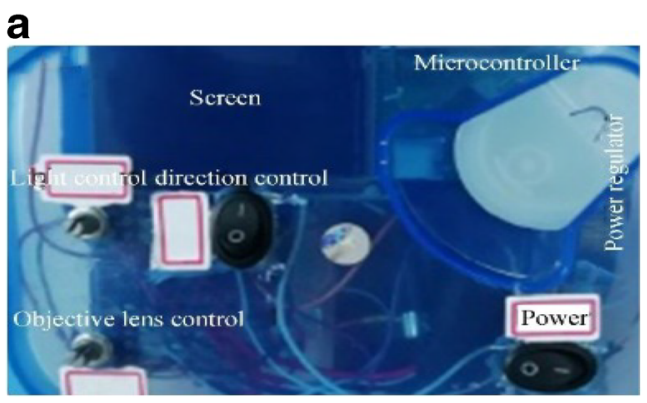

b

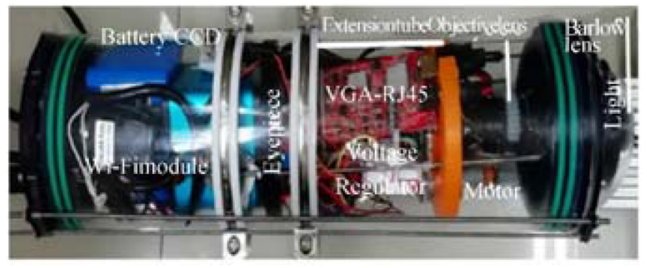

C

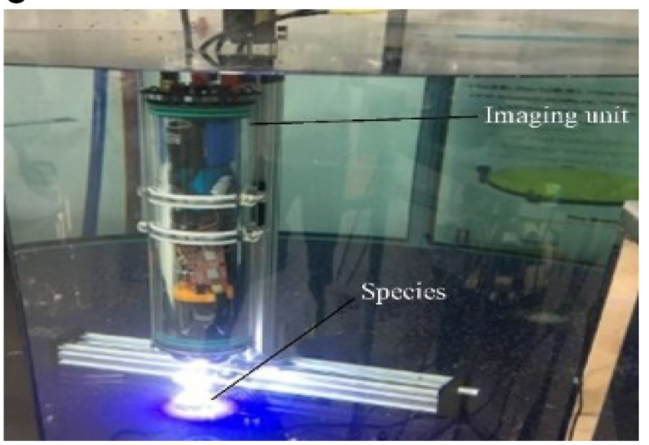

d

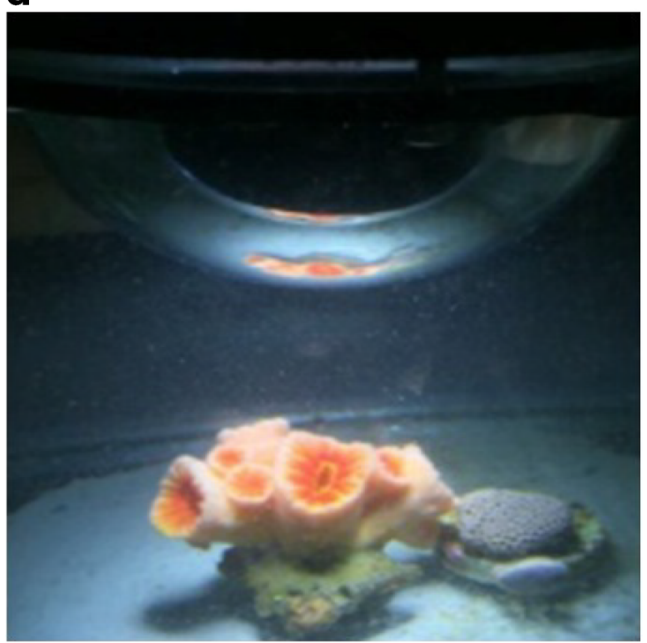

Figure 1 Description of the underwater microscope and its operation. a Control unit. b Imaging unit. c Underwater microscope in the test tank with two-axis stepper-controlled assembly. d Imaging device and coral target are $>90-\mathrm{mm}$ apart

The optical system shown in Figure 2 comprises one Lapsun (M102) CCD camera with pixel size $1 \frac{1}{2} .8$, a frame rate of $60 \mathrm{fps}$, and 14 megapixels at 1080p resolution with 2.4-23$\mathrm{cm}$ field of view at the working distance of $10 \mathrm{~cm}$; C-mount $\times$ 2 eyepiece Barlow lens; C-mount $\times 2$ extension adaptor; $\mathrm{C}$ mount $\times 0.7-4.5$ objective lens; and $\mathrm{C}$-mount $\times 2$ objective 


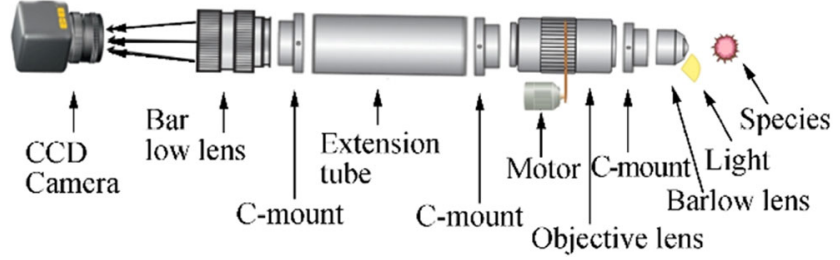

Figure 2 Optical unit of the underwater microscope

Barlow lens. The motor-controlled variable objective lens provides robust variable magnifications.

\subsubsection{Focus Stacking and Image Enhancing}

Images taken in hazy ambient scenarios are severely degraded by the dispersion of oceanic waste; moreover, hazy images have an almost poor quality because their information cannot be instantly reviewed by the human eyes (Ullah et al. 2013). In this study, contrast stretching and brightening protocols were applied to RGB images captured by the microscope to enhance the image features. Individual pixels on the image are treated rationally by darkening and brightening the cloudy and light pixels, respectively, to boost the quality of the microscopecaptured images. A raw image and the enhanced form after contrast stretching and their respective histograms are shown in Figure 3a and b. It is difficult to capture all essential parts of an image in proper focus due to changes in the focal distance. The underwater microscope can focus on a single object at a time. Thus, we could shift the focus to take various clear images of coral and later combine all the clear images using Zerene Stacker focus stacking software, as shown in Figure 3c.

\section{Results}

\subsection{Resolution Characterization}

A resolution study of the underwater microscope was performed in a laboratory testing tank containing seawater, with $32 \mathrm{ppt}$ salinity, and in air using synthetic U.S. Air Force resolution chart (USAF-1951), which is mainly used for the calibration and estimation of the microscope resolution. The USAF-1951 scale was snapped in air and testing tank by the underwater microscope, and the pixel intensity distribution of the images was revealed through observation using the histogram function of the Matlab software, as shown in Figure 4a-d.

In this study, the resolution was also quantified as line pairs per millimeter using USAF. Although the USAF test yields precise resolution values, it depends on the definition of an acceptable blending of horizontal and vertical lines of certain group elements of the USAF resolution scale. As shown in Figure 4 , element 4 of group 7 was visible using a $\times 4.5$ objective lens without blending the horizontal and vertical lines, which indicates the resolution and single-line thickness of $181 \mathrm{lp} / \mathrm{mm}$ and $2.76 \mu \mathrm{m}$, respectively.

Another relevant aspect of the resolution is the depth of field (dof) of the microscope, which depends on the wavelength of induced light, the refractive index of the medium, and numerical aperture (NA) of the objective. The dof can be calculated as:

$\operatorname{dof}=\frac{\lambda \sqrt{n^{2}-N A^{2}}}{N A^{2}}$

At $\times 4$ objective with a NA of 0.1 , wavelength $(\lambda)$ of 0.56 $\mu \mathrm{m}$, and refractive index $(n)$ of 1.362 , the dof of the underwater microscope was equal to $74.7 \mu \mathrm{m}$.

Typically, the higher the magnification power, the larger the NA and, consequently, the dof becomes smaller. In the underwater microscope, the dof can be manually changed by adjusting the objective lens magnification.

a
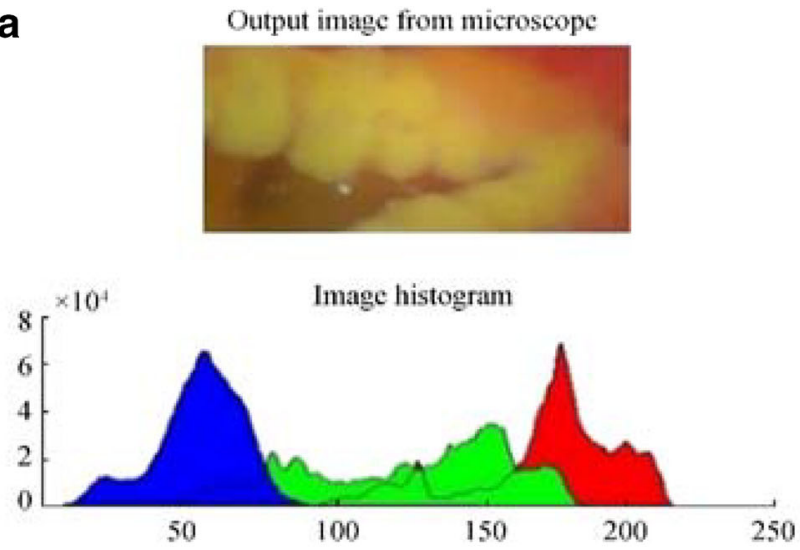

b
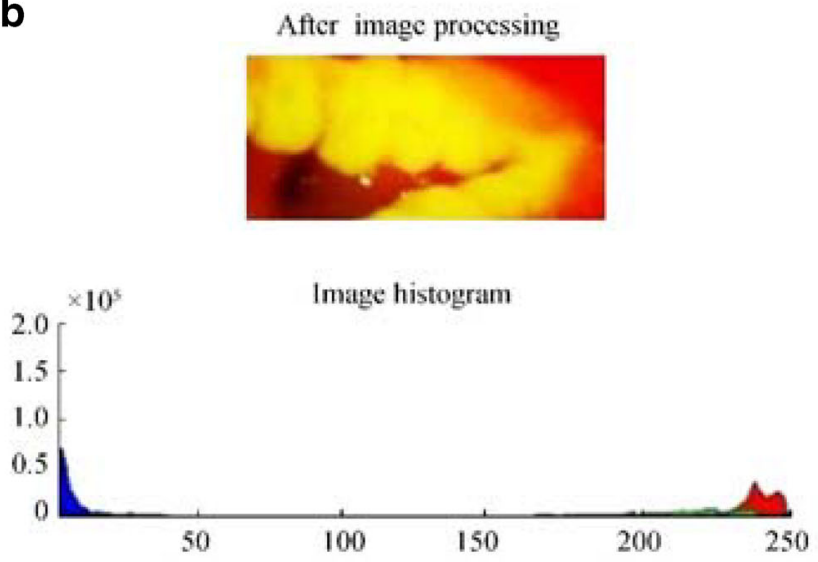

C

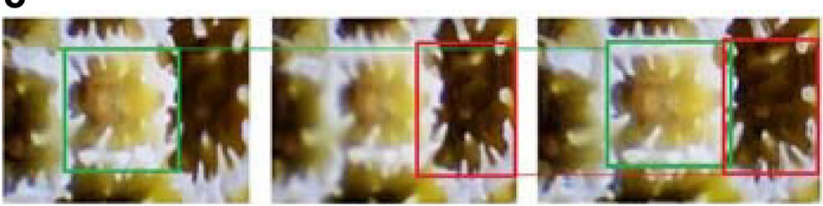

Figure 3 Image enhancement and focus stacking illustration. a Initial color image of coral in test tank. b Contrast enhancement of coral image ( $x$ and $y$ axes represents pixel intensity and pixel frequency, respectively). c Coral images at different focuses and focus stacking 
a

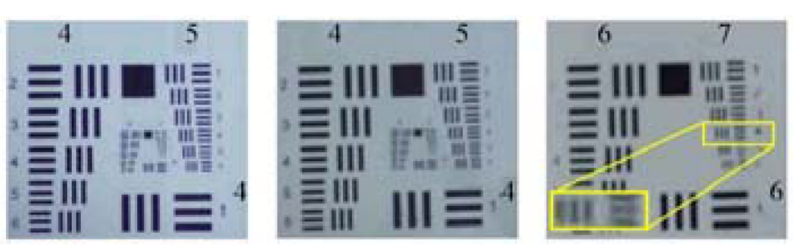

b

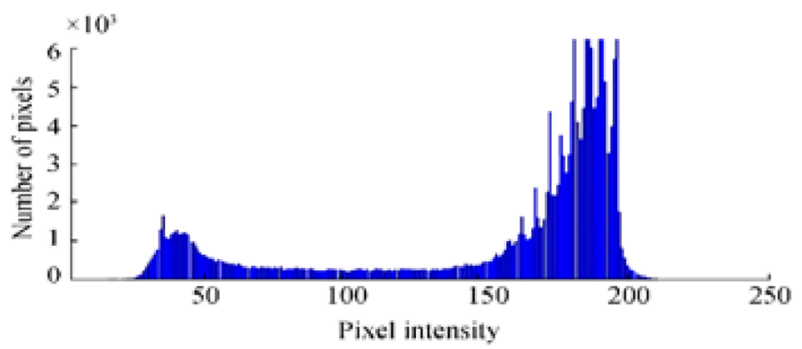

C

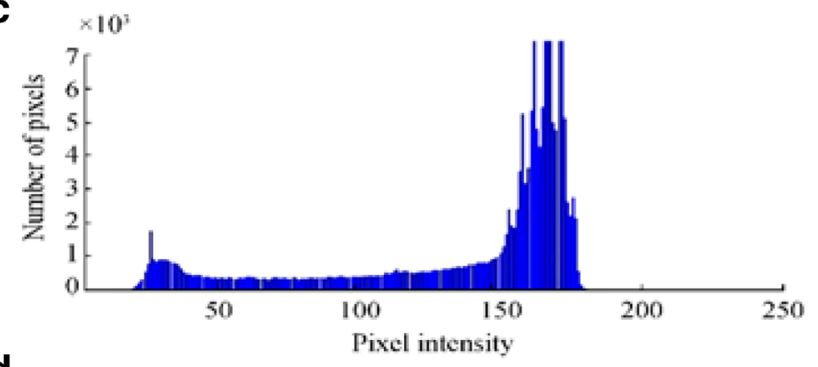

d

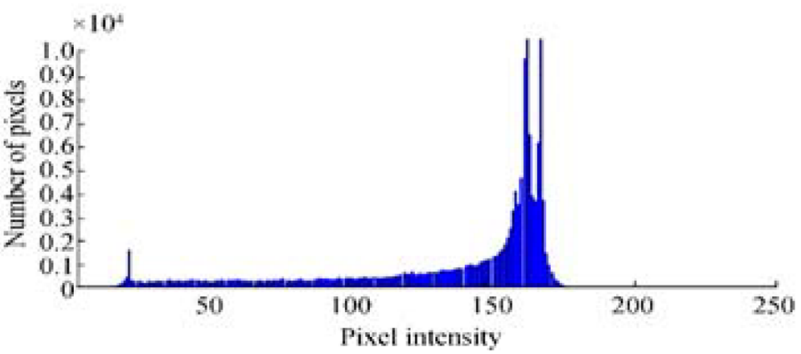

Figure 4 Resolution testing with USAF-1951 chart. a Image captured in the air at $\times 1028.4$ digital magnification with $\times 3$ objective lens, image captured with $\times 3$ objective lens in the water at $\times 1028.4$ digital magnification, and image captured with $\times 4.5$ lens in water at $\times 1542.6$ digital magnification. b Pixel intensity of the image in air with $\times 3$. c Pixel intensity of the image in water with $\times 3$. d Pixel intensity of the image in water with $\times 4.5$

\subsection{Underwater Analysis}

\subsubsection{River Reed Sediment Study}

Sediments are naturally occurring rock materials that are broken into small fragments and grains through erosion and weathering processes. They move from one place to another through erosion (Tucker 2009). Sand is an assembly of heavy minerals that are heavier than quartz and usually dark in color. This type of heavy mineral accumulation is found on beaches because of the transport of smaller particles under wave action, leaving the heavier particles behind. Hence, heavy mineral particles play a vital role in the formation of black and darker sand. The color of sand and the color of its heavy particles determine the beach color. The most common type of sand color is light brown, which emanates from iron staining in quartz and iron oxide in feldspar which are the two most common constituents of sand found on most beaches. Black-colored sand on some beaches is formed by eroded basalt lava from volcanoes. The designed microscope was used under the sea to determine the lithology, color, and grain size of sediments (Vaasma 2008). In addition, the underwater microscope can study the physical characteristics of different minerals found in heavy mineral concentrates and fine fractions recovered from streams and rivers. Based on the size grading of grains by Wentworth (1922), clay has a size of $0.06 \mu \mathrm{m}$, silt is $3.9-31 \mu \mathrm{m}$, and sand is $63-500 \mu \mathrm{m}$. For gradation, the underwater microscope was used to obtain different images of sediments in the testing tank, and the imaging performance is displayed in Figure 5a. The grain size of surface and bottom sediments can be defined from digital images using various methods (Rubin 2004). In addition, the watershed segmentation method (Rabbani and Ayatollahi 2015) was used to measure the average grain radius, which was 94.02 micron, as shown in Figure 5b.

\subsubsection{Spatial Pattern Analysis of Coral Ecology}

Spatial pattern analysis is a method for analyzing the frequency of discrete points. It is an analytical method employed to gather and analyze data on the spatial formation of individuals scattered inside an ecosystem. The underwater microscope was used to obtain spatial observation data at micro scales, thus showing its capabilities for examining the distribution patterns of corals undergoing bleaching. Coral reefs require less nutrients, and clear water permits sunlight to reach the zooxanthellae. Corals also provide shelter and nutrients to zooxanthellae for their photosynthetic activities. Moreover, corals depend on bacteria (in a kind of symbiotic association) to obtain essential compounds for their survival (Shelyakin et al. 2018). In addition, corals are also affected by slight changes in the physiochemical and environmental parameters of the ocean (Huggett and Apprill 2018).

Variation in the relationship between a coral and its microbes can cause coral disease. This alteration is due to anthropogenic impacts, including environmental change and contaminated water quality near coral reefs (Grottoli et al. 2018). This way, the coral disease progresses to bleaching. Continued spatial pattern analysis of coral reefs and coral microbiomes through the underwater microscope is important to understand the ecology and biology of corals and coral reefs and how they are changing, particularly in response to external events such as bleaching and coastal pollution (Hughes et al. 2018; Massé et al. 2018). 
In situ images acquired in a testing tank in the Optoelectronics and Automation Laboratory of Ocean College, Zhejiang University, China, showed different algal overgrowths on bleached hexagonaria coral. All images were captured within two days. The $\times 3$ and $\times 4.5$ objective lens were used to provide $3-\mathrm{mm}$ and $2-\mathrm{mm}$ field of view, respectively. The image captured from the close examination of coral polyps is shown in Figure 6a. Algal growth occurred on the surface and inside the coral. The images illustrate the locations of algae, as represented by green cells. Algal division was performed using a global saturation threshold, as in Figure $6 b$.

\subsubsection{Analysis of Zooplankton Ecology}

Plankton, also known as "drifters," is a diverse heterogeneous group of tiny organisms suspended in large water bodies such as oceans and in freshwater bodies such as lakes, ponds, streams, and rivers (Dai et al. 2017). As an indicator of water quality, plankton, including zooplankton and phytoplankton, is considered an integral component of the global carbon cycle and the foundation of the food chain for aquatic life (Blaschko et al. 2005; Zheng et al. 2017).

Zooplankton samples were collected using a Heron Tranter net from the Lake of Ocean College, Zhejiang University, Zhoushan, China. The samples were kept inside the water tank, so that the lake water could develop to become like the natural lake environment for zooplankton species. Later, the abundance and distribution of zooplankton were captured using the underwater microscope.

In this study, the major plankton species predominantly present in the Lake of Ocean College were determined and captured. The identification was performed in the Biological Ocean Department of Ocean College, Zhejiang University. A biologist observed the captured images and revealed that Copepod, Rotifer, and Daphnia, as shown in Figure 7 were dominant in the lake water. Of these, Rotifer and Copepod were present in higher numbers in the lake water; this clarifies the plankton distribution and diversity in a freshwater ecosystem. The dominant species is dependent on the lake field and the amount of phytoplankton present in it.

\subsubsection{Analysis of Microplastic}

Microplastics are plastics that are $<5 \mathrm{~mm}$ in diameter (Peng et al. 2017). They can exist in pellet, film, and fiber forms and range from microns to millimeters in size.

Microplastic is a major cause of marine pollution, as demonstrated by its distribution in aquatic water ecosystems due to industrialization and water discharge from industries and houses. Their small size allows them to enter the food web upon their uptake by marine organisms ( $\mathrm{Li}$ et al. 2016; Peng et al. 2017). Ingested particles can lead to physical damage or
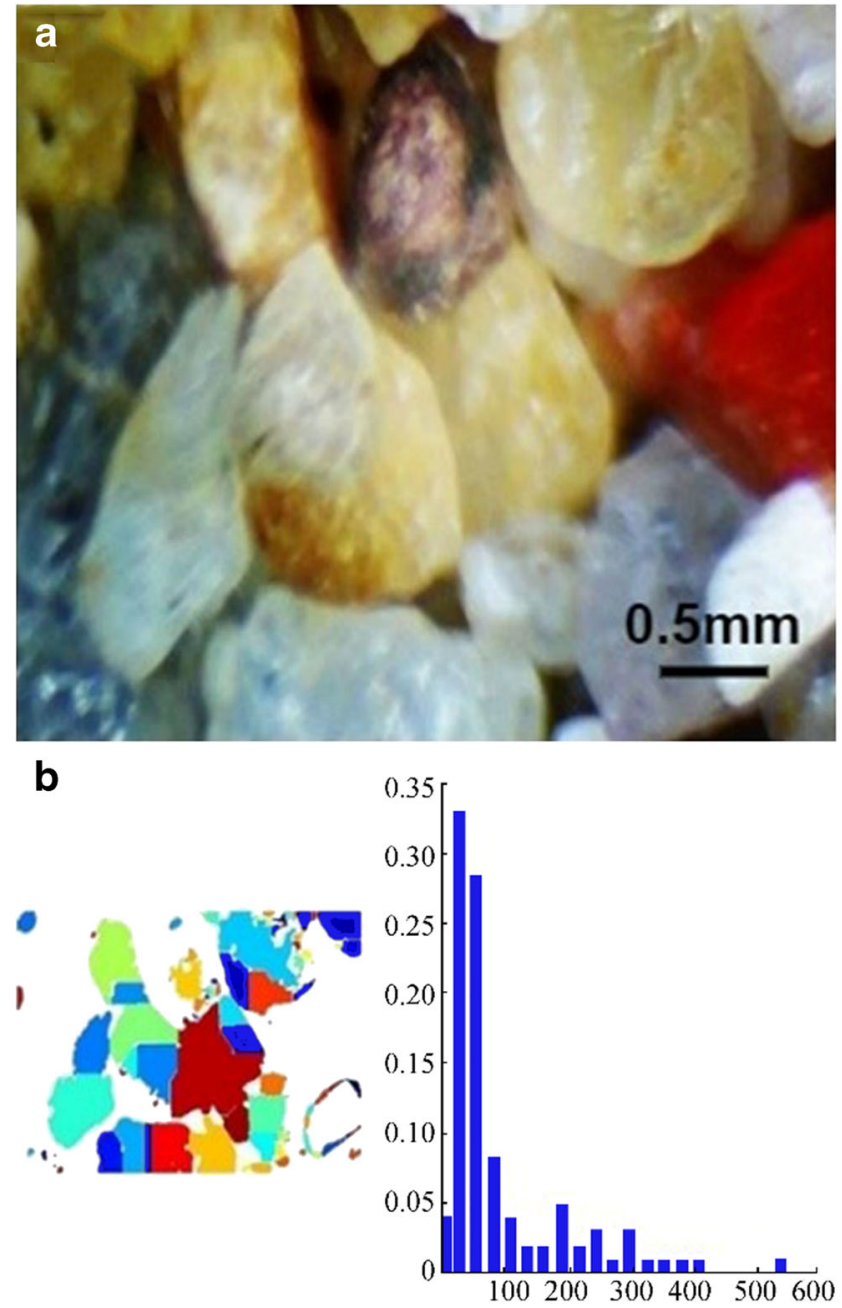

Figure 5 Underwater sediments observation with the underwater microscope. a Image of sediments taken in testing tank with $\times 3$ objective lens. b Image analysis showing the average grain radius of sediments, where $x$ and $y$ axes represent particle frequency and particle radius (micron), respectively

alter the animals' digestive system. The adverse effect of microplastic can be seen in fishes and other aquatic animals, since they are present in the guts and intestines of fishes. The bioaccumulation and biomagnification of microplastics in the food web can potentially lead to its intake by humans.

Some samples were collected from the Lake of Ocean College, Zhejiang University, Zhoushan, China, and were sieved using 5-mm sieves. Afterward, the samples were placed in our experimental water tank to capture images using the underwater microscope. To better study our designed microscope, we isolated microplastic from face wash products and cut some plastic into thin particles and obtained their images with the underwater microscope (Figure 8). The identification was performed with assistance from the Biological Oceanography Department of Ocean College, Zhejiang University Zhoushan, China. 


\section{Discussion}

The underwater microscope for in situ imaging has the advantage of an integrated motor-controlled magnification by the objective lens. The underwater microscope is lightweight and cost-effective and has no complex electronic structure. It has various advantages over traditional methods, which are labor-, cost-, and time-intensive. During the experiment, it was observed that group-7 elements of the USAF-1951 resolution scale with $2.76-\mu \mathrm{m}$ resolution were visible underwater.

This study shows that in situ microscopy using the underwater microscope is a reliable and valid method for studying corals to understand the effects of degradation and algal accumulation. Furthermore, it has been proved effective for the determination of sediment grain size, zooplankton diversity, and presence of microplastics. Sea sediments were examined by the underwater microscope, and the results showed that the underwater microscope technique can be more reliable than laboratory procedures, in which the water surface suffers little disruption, and thus, the sampling consumes extra time. The underwater microscopic images reveal the change in the physical, biological, and morphological characteristics of corals. Under observation by the underwater microscope, corals in the testing tank showed degradation signs after some days. Further observations of damaged corals indicated that polyps and their associated microorganisms were not present, since most of the corals were covered by algae. The presence of algae indicates coral bleaching, which is the norm for corals living in poor nutrient-conditions (Serrano et al. 2018).

Besides identifying the algae micro-scale spatial patterns, images were further examined by applying the global saturation threshold technique using Matlab. Based on our results of the underwater microscope efficiency experiment, the system is accurate, practical, and well-functional, and it can be modified to examine benthic communities and clarify the aquatic ecosystems. Moreover, the results show that the underwater microscope can store time-series images at different magnifications, and these images can be later processed to examine particular underwater conditions. The underwater microscope provides the ability to capture images in benthic aquatic ecosystems, thus enabling the observations of biological and ecological variations. Previous studies have demonstrated the need for a tool for observing benthic aquatic communities; the novel underwater microscope can capture images at various magnifications (Mullen et al. 2016; Rubin et al. 2007). Furthermore, underwater microscopy can clarify the interactions in natural aquatic ecosystems. Anthropogenic activities, industrialization, and the discharge of untreated effluents into the aquatic ecosystem lead to coral degradation and bleaching (Jafarabadi et al. 2018). The underwater microscope can provide new approaches to aquatic ecosystem observation, especially on algae-coral relationship, coral bleaching and improvement, disease study, and larval arrangement and growth. As a resourceful device, the underwater microscope has clear and practical applications in an extensive variety of scientific fields, including marine ecology, marine geology, physiology, and biomechanics. The developed microscope design and suitability make the underwater microscope useful for aquatic ecosystem imaging and suitable for laboratory and ocean research. Compared with laboratory methods, real-time underwater microscopy is more reliable, since, in laboratory research, the sampling disturbs the aquatic ecosystem (Mullen et al. 2016; Rubin et al. 2007).

The new underwater microscope overcomes the significant challenges presented by Mullen et al. (2016) and Rubin et al. (2007), as it has integrated variable objective lenses, and realtime images are directly acquired on the surface controlling unit. The microscope deployed on a two-axis stepper-controlled linear assembly provides greater stability and focus in aquatic conditions.
Figure 6 Study of corals with the underwater microscope. a The first two images captured with $\times 3$ when coral is alive. The last two images captured with $\times 4.5$ when coral is dead. b Saturation images where green color represents algae
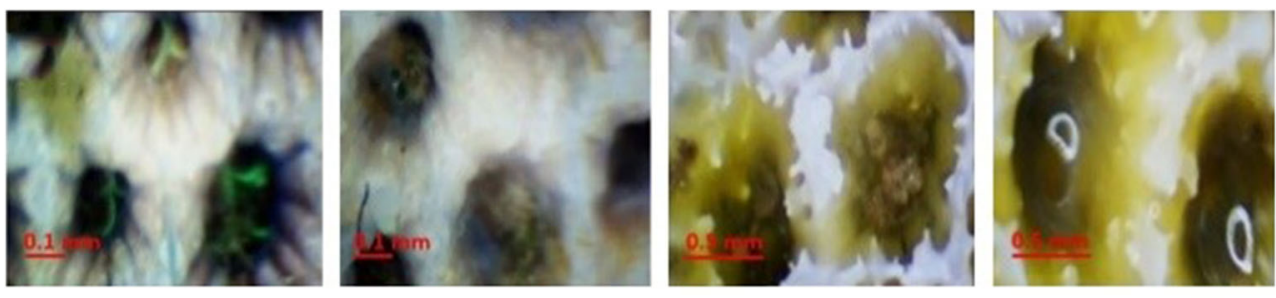

a
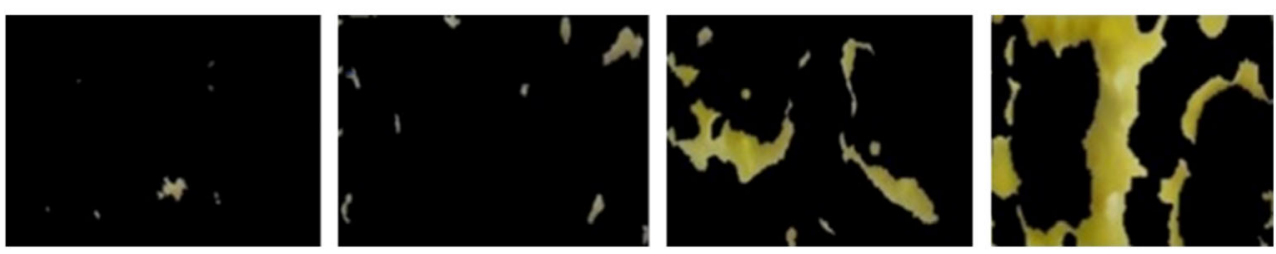

b 

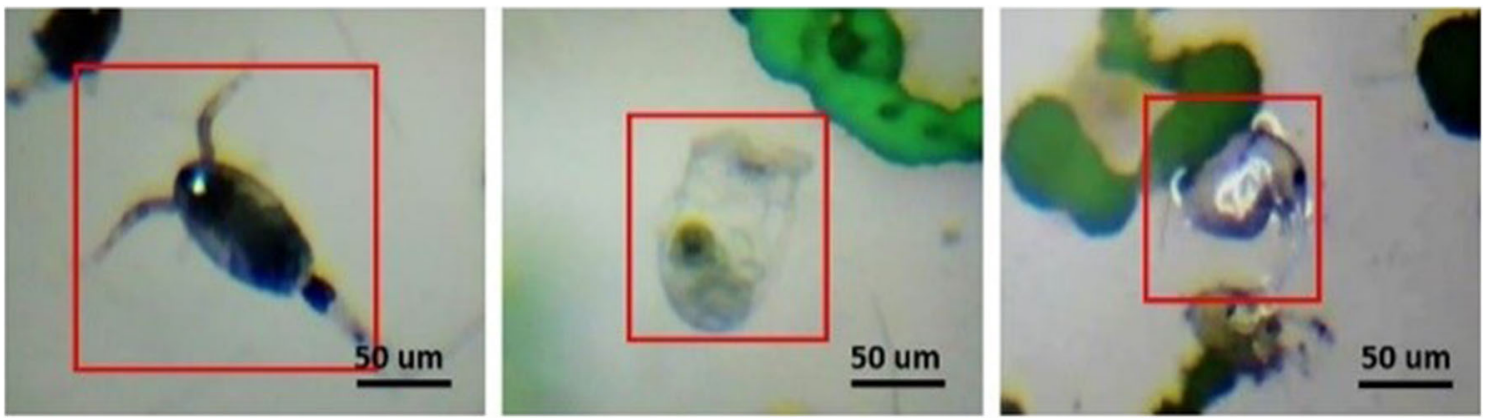

Figure 7 Zooplankton micro-images captured by the underwater microscope with $\times 1.5$ objective lens showing the presence of Copepod, Rotifer, and Daphnia. Green color shows the presence of algae

\section{Conclusions}

Our trial experiments showed that the underwater microscope can be used for the in situ microscopic imaging of benthic communities to monitor coral ecology, sediment lithology and grain size, zooplanktons, and microplastics at different magnifications. It is useful for ecosystem analysis; the study of the effects of associated microbes, growth, and degradation; and coral bleaching, thus clarifying interactions at the micro-level.
It is an innovative tool that can enable researchers to effectively capture images for the observation and classification of the benthic ecosystems. The approach also has certain limitations, which will be the subject of future research and improvement. For example, as a future perspective, an automated classification technique of the underwater microscope would be essential to identify and classify the benthic ecosystem. We will also try to attach a wireless link from the controller to the laboratory for real-time access.
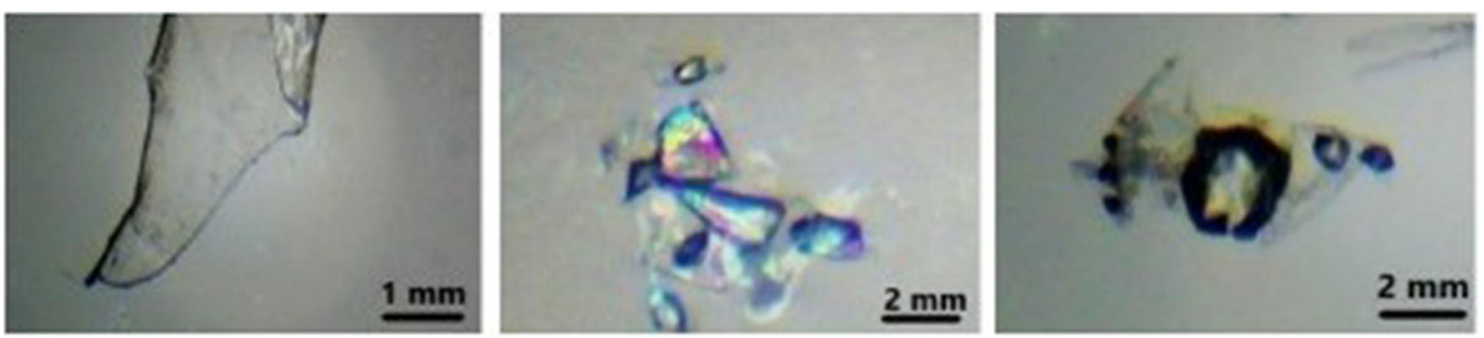

a
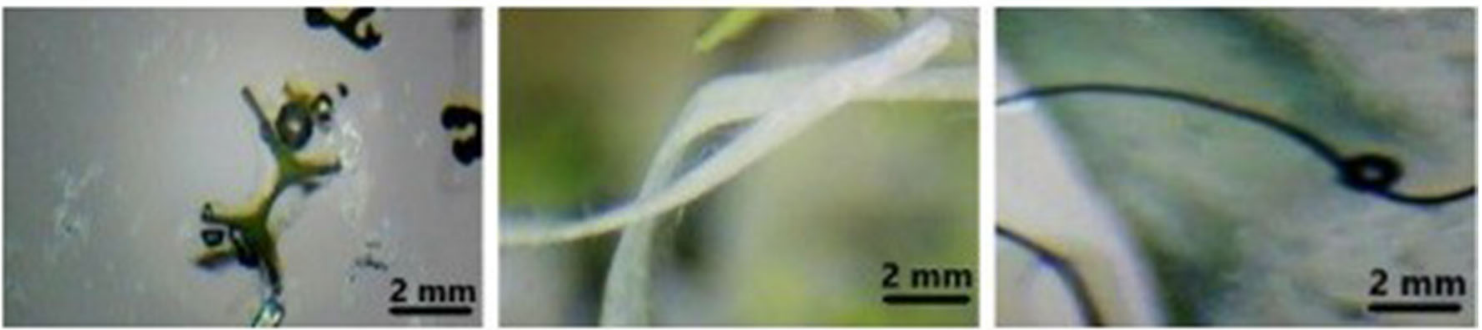

b
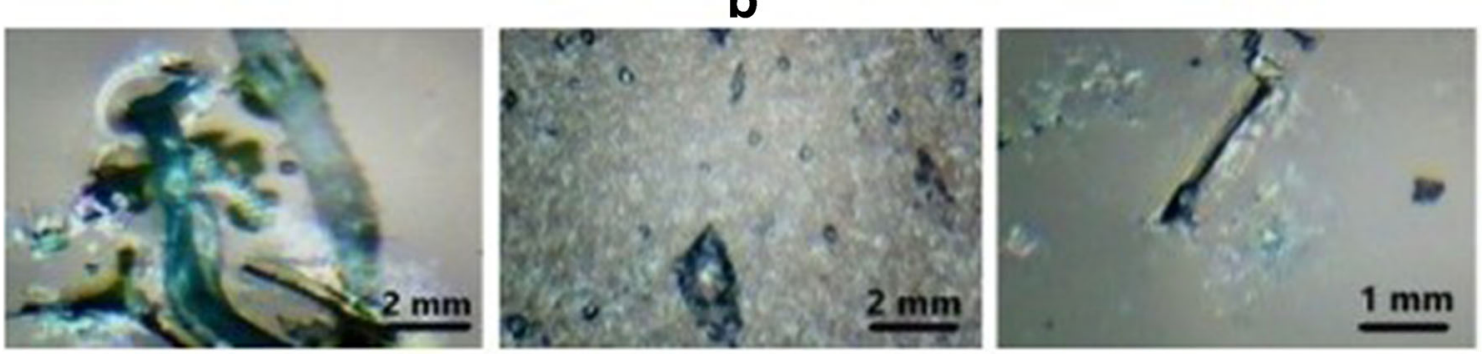

C

Figure 8 Images captured by underwater microscope in testing tank explaining the size and forms of microplastics. a Microplastic films. b Microplastic fibers. c Microplastic beads 
Acknowledgments The authors would like to express sincere gratitude to Chaopeng Wu, Yichun Shentu, and Yilu Guo from the Smart Ocean Optics Lab for their support in the development of the system.

Funding This study is supported by the Key Research and Development Plan of Zhejiang Province, China (Grant number: 2020C03012).

Open Access This article is licensed under a Creative Commons Attribution 4.0 International License, which permits use, sharing, adaptation, distribution and reproduction in any medium or format, as long as you give appropriate credit to the original author(s) and the source, provide a link to the Creative Commons licence, and indicate if changes were made. The images or other third party material in this article are included in the article's Creative Commons licence, unless indicated otherwise in a credit line to the material. If material is not included in the article's Creative Commons licence and your intended use is not permitted by statutory regulation or exceeds the permitted use, you will need to obtain permission directly from the copyright holder. To view a copy of this licence, visit http://creativecommons.org/licenses/by/4.0/.

\section{References}

Akiba T, Kakui Y (2000) Design and testing of an underwater microscope and image processing system for the study of zooplankton distribution. IEEE J Ocean Eng 25(1):97-104. https://doi.org/10. $1109 / 48.820741$

Arora M, Sahoo D (2015) Concepts and techniques for the study of algae. In: The Algae World. Springer, Dordrecht. https://doi.org/10.1007/ 978-94-017-7321-8 21

Ateweberhan M, Feary DA, Feshavmurthy S, Chen A, Schleyer MH, Sheppard CR (2013) Climate change impacts on coral reefs: synergies with local effects, possibilities for acclimation, and management implications. Mar Pollut Bull 74(2):526-539. https://doi.org/ 10.1016/j.marpolbul.2013.06.011

Blaschko MB, Holness G, Mattar MA, Lisin D, Utgoff PE, Hanson AR, Schultz H, Riseman EM, Sieracki ME, Balch WM, Tupper B (2005) Automatic in situ identification of plankton. 7th IEEE Workshop on Applications of Computer Vision 1, 79-86. https://doi.org/10.1109/ ACVMOT.2005.29

Dai J, Yu Z, Zheng H, Zheng B, Wang N (2017) A hybrid convolutional neural network for plankton classification. 2016 Asian Conference on Vision, 102-114. https://doi.org/10.1007/978-3-319-54526-4_8

Delgadillo-Nuño MA, Liñán-Cabello MA, Reyes-Gómez J, SoriañoSantiago O (2014) Response to $\mathrm{pH}$ stress in the reef-building coral Pocillopora capitata (Anthozoa: Scleractinia). Rev Biol Mar Oceanogr 49(3):449-459. https://doi.org/10.4067/S071819572014000300004

Gao S, Collins MB (1994) Analysis of grain size trends, for defining sediment transport pathways in marine environments. J Coast Res 10(1):70-78 https://www.jstor.org/stable/4298194

Grottoli AG, Martins PD, Wilkins MJ, Johnston MD, Warner ME, Cai WJ, Melman TF, Hoadley KD, Pettay DT, Levas SJ (2018) Coral physiology and microbiome dynamics under combined warming and ocean acidification. PLoS One 13(1):e0191156. https://doi. org/10.1371/journal.pone.0191156

Huggett MJ, Apprill AJ (2018) Coral microbiome database: integration of sequences reveals high diversity and relatedness of coral-associated microbes. Environ Microbiol Rep 11(3):372-385. https://doi.org/ $10.1111 / 1758-2229.12686$
Hughes TP, Anderson KD, Connolly SR, Heron SF, Kerry JT, Lough JM, Baird AH, Baum JK, Berumen ML, Bridge TC (2018) Spatial and temporal patterns of mass bleaching of corals in the Anthropocene. Science 359(6371):80-83. https://doi.org/10.1126/ science.aan 8048

Jafarabadi AR, Bakhtiari AR, Hedouin L, Toosi AS, Cappello T (2018) Spatio-temporal variability, distribution and sources of $n$-alkanes and polycyclic aromatic hydrocarbons in reef surface sediments of Kharg and Lark coral reefs, Persian Gulf, Iran. Ecotoxicol Environ Saf 163:307-322. https://doi.org/10.1016/j.ecoenv.2018.07.056

Jaffe JS, Moore KD, Mclean J, Strand MP (2001) Underwater optical imaging: status and prospects. Oceanography 14(3):66-76. https:// doi.org/10.5670/oceanog.2001.24

Kennelly SJ, Underwood AJ (1984) Underwater microscopic sampling of a sublittoral kelp community. J Exp Mar Biol Ecol 76(1):67-78. https://doi.org/10.1016/0022-0981(84)90017-0

Li J, Qu X, Su L, Zhang W, Yang D, Kolandhasamy P, Li D, Shi H (2016) Microplastics in mussels along the coastal waters of China. Environ Pollut 214:177-184. https://doi.org/10.1016/j.envpol.2016. 04.012

Massé A, Domart-coulon I, Golubic S, Duché D, Tribollet A (2018) Early skeletal colonization of the coral holobiont by the microboring Ulvophyceae Ostreobium sp. Sci Rep 8(1):1-11. https://doi.org/ 10.1038/s41598-018-20196-5

Mccook L, Jompa J, Diaz-pulido G (2001) Competition between corals and algae on coral reefs: a review of evidence and mechanisms. Coral Reefs 19(4):400-417. https://doi.org/10.1007/ s003380000129

Mullen AD, Treibitz T, Roberts PL, Kelly EL, Horwitz R, Smith JE, Jaffe JS (2016) Underwater microscopy for in situ studies of benthic ecosystems. Nat Commun 7(1):1-9. https://doi.org/10.1038/ ncomms 12093

Neushul M (1972) Underwater microscopy with an encased incident-light dipping-cone microscope. J Microsc 95(3):421-424. https://doi.org/ 10.1111/j.1365-2818.1972.tb01043.x

Peng G, Zhu B, Yang D, Su L, Shi H, Li D (2017) Microplastics in Sediments of the Changjiang Estuary, China. Environ Pollut 225: 283-290. https://doi.org/10.1016/j.envpol.2016.12.064

Rabbani A, Ayatollahi S (2015) Comparing three image processing algorithms to estimate the grain-size distribution of porous rocks from binary 2D images and sensitivity analysis of the grain overlapping degree. Spec Top Rev Porous Media 6(1):71-89. https://doi.org/10. 1615/SpecialTopicsRevPorousMedia.v6.i1.60

Rubin DM (2004) A simple autocorrelation algorithm for determining grain size from digital images of sediment. J Sediment Res 74(1): 160-165. https://doi.org/10.1306/052203740160

Rubin DM, Chezar H, Harney JN, Topping DJ, Melis TS, Sherwood CR (2007) Underwater microscope for measuring spatial and temporal changes in bed-sediment grain size. Sediment Geol 202(3):402-408. https://doi.org/10.1016/j.sedgeo.2007.03.020

Schutte VG, Selig ER, Bruno JF (2010) Regional spatio-temporal trends in Caribbean coral reef benthic communities. Mar Ecol Prog Ser 402(1):115-122. https://doi.org/10.3354/meps08438

Serrano XM, Miller MW, Hendee JC, Jensen BA, Gapayao JZ, Pasparakis C, Grosell M, Baker AC (2018) Effects of thermal stress and nitrate enrichment on the larval performance of two Caribbean reef corals. Coral Reefs 37(1):173-182. https://doi.org/10.1007/ s00338-017-1645-y

Shelyakin PV, Garushyants SK, Nikitin MA, Mudrova SV, Berumen M, Speksnijder AG, Hoeksema BW, Fontaneto D, Gelfand MS, Ivanenko VN (2018) Microbiomes of gall-inducing copepod crustaceans from the corals Stylophora pistillata (Scleractinia) and 
Gorgonia ventalina (Alcyonacea). Sci Rep 8(1):1-10. https://doi. org/10.1038/s41598-018-29953-y

Talapatra S, Hong J, Mcfarland M, Nayak AR, Zhang C, Katz J, Sullivan J, Twardowski M, Rines J, Donaghay PJ (2013) Characterization of biophysical interactions in the water column using in situ digital holography. Mar Ecol Prog Ser 473:29-51. https://doi.org/10. 3354/meps 10049

Tucker ME (2009) Sedimentary petrology: an introduction to the origin of sedimentary rocks. John Wiley \& Sons

Ullah E, Nawaz R, Iqbal J (2013) Single image haze removal using improved dark channel prior. 5th International Conference on Modelling, Identification and Control (ICMIC), IEEE, Cairo, 245-
248. http://ieeexplore.ieee.org/stamp/stamp.jsp?tp=\&arnumber= 6642199\&isnumber $=6642159$

Vaasma T (2008) Grain-size analysis of lacustrine sediments: a comparison of pre-treatment methods. Estonian J Ecol 57(4):231-243. https://doi.org/10.3176/eco.2008.4.01

Wentworth CK (1922) A scale of grade and class terms for clastic sediments. J Geol 30(5):377-392. https://doi.org/10.1086/622910

Zheng H, Wang R, Yu Z, Wang N, Gu Z, Zheng B (2017) Automatic plankton image classification combining multiple view features via multiple kernel learning. BMC Bioinf 18(16):570: 1-18. https://doi. org/10.1186/s12859-017-1954-8 